\title{
New Mixed Solutions Generated By Velocity Resonance In The (2+1)-Dimensional Sawada- Kotera Equation
}

\section{Zequn Qi}

Ningbo University

Qingqing Chen

Ningbo University

Miaomiao Wang

Ningbo University

Biao Li ( $D$ libiao@nbu.edu.cn )

Ningbo University https://orcid.org/0000-0001-9218-8837

\section{Research Article}

Keywords: Velocity resonance, Mixed solutions, Soliton molecules, Lump wave

Posted Date: November 30th, 2021

DOI: https://doi.org/10.21203/rs.3.rs-1096252/v1

License: (9) This work is licensed under a Creative Commons Attribution 4.0 International License. Read Full License

Version of Record: A version of this preprint was published at Nonlinear Dynamics on February 12th, 2022. See the published version at https://doi.org/10.1007/s11071-022-07248-2. 


\title{
New mixed solutions generated by velocity resonance in the $(2+1)$-dimensional Sawada-Kotera equation
}

\author{
Zequn $\mathrm{Qi}^{\mathrm{a}}$, Qingqing Chen ${ }^{\mathrm{a}}$, Miaomiao Wang ${ }^{\mathrm{a},}$, Biao Li ${ }^{\mathrm{a}, *}$ \\ ${ }^{a}$ School of Mathematics and Statistics, Ningbo University, Ningbo 315211, P. R. China
}

\begin{abstract}
Based on the mixed solutions of the $(2+1)$-dimensional Sawada-Kotera equation, the collisions among lump waves, line waves, and breather waves are studied in this paper. By introducing new constraints, the lump wave does not collide with other waves forever. Under the condition of velocity resonance, the soliton molecules consisting of a lump wave, a line wave and any number of breather waves are derived for the first time. In particular, the interaction of a line wave and a breather wave will generate two breathers under certain conditions, which is very interesting. Additionally, the method can also be extended to other $(2+1)$-dimensional integrable equations.
\end{abstract}

Keywords: Velocity resonance; Mixed solutions; Soliton molecules; Lump wave.

PACS: 05.45.Yv, 02.30.Ik, 47.20.Ky, 52.35.Mw

\section{Introduction}

Soliton molecules are a hot topic and have been studied extensively in recent years [1-9]. We already know that the soliton molecules are the stable bound state of solitons generated by velocity resonance [7], which breaks the previous understanding of the nature of traveling wave motion, that is, the speed of high wave is slower than the short wave. Soliton molecules can appear in optical systems or fluid systems, and can be used in many fields, including ocean surface waves, electromagnetic waves in discrete transmission lines and plasma waves, etc. It is currently known that multiple soliton solutions can be derived by the Hirota bilinear method [3,4] and the Darboux transformation method [5, 8, 11, 12], and then soliton molecules can be obtained by constraints on parameters. The mixed solutions of lump waves and other waves under velocity resonance are rarely studied, these solutions deserve further study.

It is known that the collision between two solitary waves is elastic in the soliton theory, and once a collision occurs, the movement of solitary waves follows certain rules. Therefore, this paper mainly uses bilinear method to study the novel mixed solutions of lump waves and other waves, such as a lump wave and line waves never collide, a lump wave and breathers never collide, and it is worth mentioning that these solutions can appear in many nonlinear wave equations.

We consider the $(2+1)$-dimensional Sawada-Kotera equation:

$$
u_{t}+u_{x x x x x}+15 u_{x} u_{x x}+15 u_{x} u_{x x x}+45 u^{2} u_{x}+5 u_{x x y}+15 u_{u} u_{y}+15 u_{x} \int u_{y} d x-5 \int u_{y y} d x=0
$$

\footnotetext{
* School of Mathematics and Statistics, Ningbo University, Ningbo 315211, P. R.China

Email address: libiao@nbu.edu.cn (Biao Li)
} 
Where $u=u(x, y, t)$, the equation was introduced by Konopelchenko and Dubrovsky firstly [13], and it is a significant equation in integrable systems, this equation can be further regarded as an integrable extension of the (1+1)-dimensional Sawada-Kotera equation and it may appears in various physical fields such as shallow water, ion acoustic waves in plasma and others, its research is beneficial to nonlinear science. Many scholars have conducted in-depth research on the $(2+1)$-dimensional Sawada-Kotera equation [8, 17-27], such as its soliton molecules, Y-type resonance soliton, and so on.

The main point of this paper is as follows: First, using the Hirota bilinear method to obtain the form of the $N$-soliton solutions of the $(2+1)$-dimensional Sawada-Kotera equation; Next, summarizing the velocity formulas of lump waves, line waves and breathers respectively; Finally, proposing different velocity constraints to obtain lump molecules, breather molecules and the nonlinear superposition of a line wave, a lump wave, and breathers at the same speed.

Substituting the variable transformation $u=2(\ln f)_{x x}$ into the equation Eq.(1), it is usually simplified to the following bilinear form:

$$
\left(D_{x} D_{t}+D_{x}^{6}+5 D_{x}^{3} D_{y}-5 D_{y}^{2}\right)(f \cdot f)=0
$$

Where $\mathrm{D}$ is the famous Hirota bilinear derivative operator, the $N$-soliton solutions have the form of $f_{N}$ as follows:

$$
f_{N}=\sum_{\mu=0,1} \exp \left(\sum_{j<s}^{N} \mu_{j} \mu_{s} A_{j s}+\sum_{j=1}^{N} \mu_{j} \xi_{j}\right),
$$

with

$$
\xi_{j}=k_{j} x+w_{j} t+p_{j} y+\phi_{j}, \quad k_{j}^{6}+5 k_{j}^{3} p_{j}-5 p_{j}^{2}+w_{j} k_{j}=0
$$

and the $e^{A_{j s}}$ is

$$
\begin{aligned}
& \left\{k_{1}{ }^{6} k_{2}{ }^{2}-3 k_{1}{ }^{5} k_{2}{ }^{3}+\left(4 k_{2}{ }^{4}+p_{2} k_{2}\right) k_{1}{ }^{4}-3\left(k_{2}{ }^{3}-2 / 3 p_{1}+p_{2}\right) k_{2}{ }^{2} k_{1}{ }^{3}+\left(k_{2}{ }^{6}+\left(-3 p_{1}+2 p_{2}\right) k_{2}{ }^{3}+p_{2}{ }^{2}\right)\right. \\
& \left.k_{1}{ }^{2}+k_{2} p_{1}\left(k_{2}{ }^{3}-2 p_{2}\right) k_{1}+k_{2}{ }^{2} p_{1}{ }^{2}\right\}\left\{k_{1}{ }^{6} k_{2}{ }^{2}+3 k_{1}{ }^{5} k_{2}{ }^{3}+\left(4 k_{2}{ }^{4}+p_{2} k_{2}\right) k_{1}{ }^{4}+3 k_{2}{ }^{2}\left(k_{2}{ }^{3}+2 / 3 p_{1}+p_{2}\right) k_{1}{ }^{3}+\right. \\
& \left.\left(k_{2}{ }^{6}+\left(3 p_{1}+2 p_{2}\right) k_{2}{ }^{3}+p_{2}{ }^{2}\right) k_{1}{ }^{2}+k_{2} p_{1}\left(k_{2}{ }^{3}-2 p_{2}\right) k_{1}+k_{2}{ }^{2} p_{1}{ }^{2}\right\}^{-1} .
\end{aligned}
$$

Based on the $N$-soliton solutions, a new type of mixed solutions is derived using the partial long wave limit method and the new constraints mentioned in this paper, in which the lump wave will never collide with other waves or always collide with other waves. The discovery of this new type of nonlinear superposition will enrich the interaction solutions in fluid systems. In particular, the trajectories of lump wave before and after its collision with other waves have been studied in some articles [14-17].

It is known that the orthogonal decomposition method can be used to study the uniform motion of objects, so the motion properties of solitons can also be studied by this method. The soliton moves at a uniform speed in space with the change of time, so it can be regarded as moving in two mutually perpendicular directions, which represent the speed in the $\mathrm{x}$ direction and the $\mathrm{y}$ direction, respectively. According to previous research [10], the velocity formulas of line waves and breathers can be get through calculation:

$$
V_{\text {line }}=\left[\text { line }_{1}, \text { line }_{2}\right]^{\mathrm{T}}=\left[-\frac{w_{s} k_{s}}{k_{s}{ }^{2}+p_{s}{ }^{2}},-\frac{w_{s} p_{s}}{k_{s}{ }^{2}+p_{s}{ }^{2}}\right]^{\mathrm{T}}
$$




$$
V_{\text {breather }}=\left[\text { breather }_{1}, \text { breather }_{2}\right]^{\mathrm{T}}=\left[-\frac{\mathfrak{R}\left(w_{s}\right) \mathfrak{R}\left(k_{s}\right)}{\left(\mathfrak{R}\left(k_{s}\right)\right)^{2}+\left(\mathfrak{R}\left(p_{s}\right)\right)^{2}},-\frac{\mathfrak{R}\left(w_{s}\right) \mathfrak{R}\left(p_{s}\right)}{\left(\mathfrak{R}\left(k_{s}\right)\right)^{2}+\left(\mathfrak{R}\left(p_{s}\right)\right)^{2}}\right]^{\mathrm{T}} .
$$

Lump wave can be obtained by long wave limit method and module resonance [10, 14-16], and based on formula Eq.(3), let $N=2, k_{1}=K_{1} * \epsilon, k_{2}=K_{2} * \epsilon, p_{1}=P_{1} * \epsilon, p_{2}=P_{2} * \epsilon, \phi_{1}=\phi_{2}=\pi * i, K_{1}=K_{2}^{*}, P_{1}=P_{2}^{*}, \epsilon \rightarrow 0$. the velocity of a lump wave can be expressed in the following form:

$$
V_{\text {lump }}=\left[\text { lump }_{1}, \text { lump }_{2}\right]^{\mathrm{T}}=\left[\frac{5 P_{1} P_{2}}{K_{1} K_{2}}, \frac{-5\left(K_{1} P_{2}+K_{2} P_{1}\right)}{K_{1} K_{2}}\right]^{\mathrm{T}} .
$$

\section{The new nonlinear superposition of a lump wave and line waves}

In this section, new mixed solutions of a lump wave and line waves are obtained by putting forward a new constraint in Eq.(3). There are two situations, the first is that a lump wave moves along the line wave without collision or always keeps colliding, and the other is that after introducing velocity resonance, a lump wave and a line wave move in the same direction at the same speed. In particular, it has been verified that the velocity resonance among a lump and two line waves does not exist.

Proposition 1: On the basis of the $N$-soliton solutions, if the 3-soliton solutions satisfy the partial long wave limit constraints, the new nonlinear superposition of a lump wave and line waves can be derived through the following constraints:

$$
k_{1}=K_{1} \epsilon, k_{2}=K_{2} \epsilon, p_{1}=P_{1} \epsilon, p_{2}=P_{2} \epsilon, \phi_{1}=\pi i, K_{1}=K_{2}^{*}, P_{1}=P_{2}^{*}, \epsilon \rightarrow 0 .
$$

(i) Lump and line waves never collide or remain collided:

$$
\lambda_{3}==-\frac{k_{3}{ }^{6}+5 k_{3}{ }^{3} p_{3}-5 p_{3}{ }^{2}}{k_{3}}+\frac{5 P_{1} P_{2} k_{3}}{K_{1} K_{2}}-\frac{5\left(K_{1} P_{2}+K_{2} P_{1}\right) p_{3}}{K_{1} K_{2}}=0 .
$$

(ii) The velocity resonance of a lump wave and a line wave:

$$
\left[\frac{5 P_{1} P_{2}}{K_{1} K_{2}}, \frac{-5\left(K_{1} P_{2}+K_{2} P_{1}\right)}{K_{1} K_{2}}\right]^{\mathrm{T}}=\left[-\frac{w_{s} k_{s}}{k_{s}^{2}+p_{s}^{2}},-\frac{w_{s} p_{s}}{k_{s}^{2}+p_{s}^{2}}\right]^{\mathrm{T}} .
$$

The mixed solutions represented by Proposition 1 include two cases. The first one is that the motion trajectory of the lump wave is parallel or coincident with the line wave, simply adjusting the phase of the line or lump wave can control whether they collide or not. A lump wave moves along the line wave as shown in Fig.(1), and the double-peaked lump wave appears. Fig.(2) shows that the lump moves over time but never collides with the line wave. In particular, we found that a lump and two line waves also produce the never-colliding situation and it is shown in Fig.(3), in this way, we can continue to explore a lump and $\mathrm{N}$ line waves never collide. The second case is that the lump wave and the line wave can be seen as a whole, which is also called lump-line molecules, moving in a certain direction together, this phenomenon can be observed in Fig.(4).

In the first type of interaction solution in Proposition 1, the trajectory of the lump wave is parallel to a straight line

$$
x=\frac{5 P_{1} P_{2} t}{K_{1} K_{2}}+l_{1}, y=\frac{-5\left(K_{1} P_{2}+K_{2} P_{1}\right) t}{K_{1} K_{2}}+l_{2} .
$$

Where $|t|$ is large enough and $l_{1}, l_{2}$ are real parameters, and Eq.(3) can be expressed as 


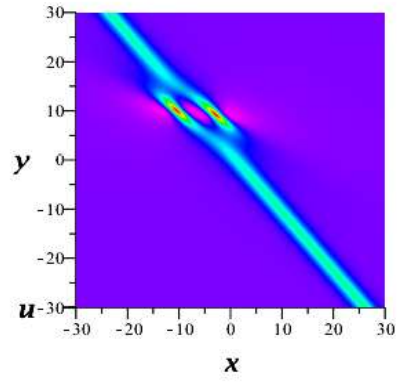

(a) $t=-1$

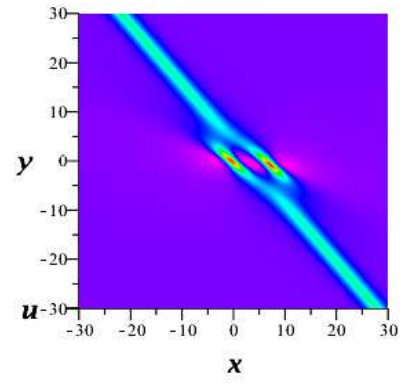

(b) $\mathrm{t}=0$

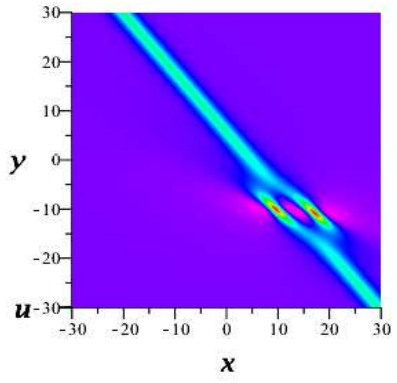

(c) $\mathrm{t}=1$

Figure 1: (Color online) A lump wave and a line wave always collide described by Proposition 1 with the parameters $\left\{N=3, K_{1}=1+i, K_{2}=1-i, P_{1}=\right.$ $\left.2, P_{2}=2, k_{3}=1, p_{3}=\frac{3}{2}-\frac{3, \sqrt{5}}{10}, \phi_{1}=i \pi, \phi_{2}=i \pi, \phi_{3}=-3\right\}$.

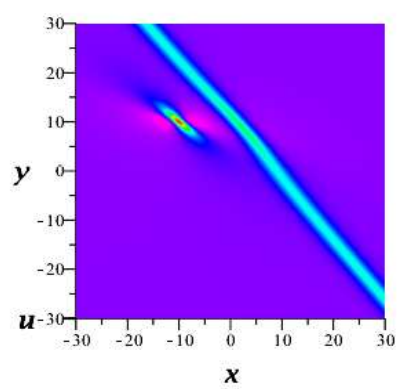

(a) $t=-1$

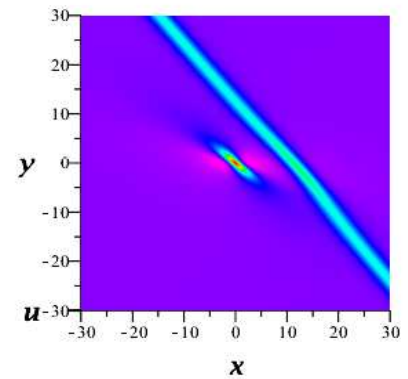

(b) $\mathrm{t}=0$

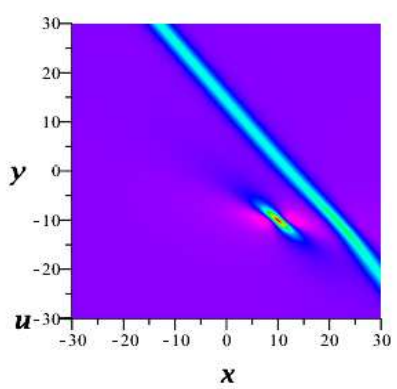

(c) $\mathrm{t}=1$

Figure 2: (Color online) A lump wave and a line wave never collide described by Proposition 1 with the parameters $\left\{N=3, K_{1}=1+i, K_{2}=1-i, P_{1}=\right.$ $\left.2, P_{2}=2, k_{3}=1, p_{3}=\frac{3}{2}-\frac{3, \sqrt{5}}{10}, \phi_{1}=i \pi, \phi_{2}=i \pi, \phi_{3}=-10\right\}$.

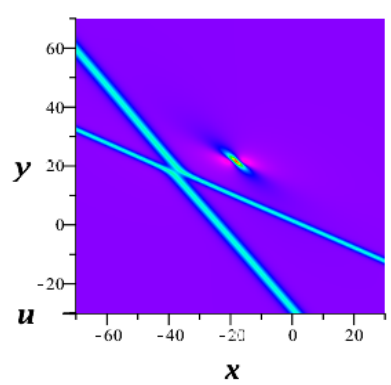

(a) $t=-2$

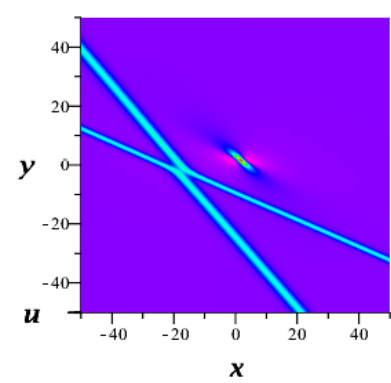

(b) $\mathrm{t}=0$

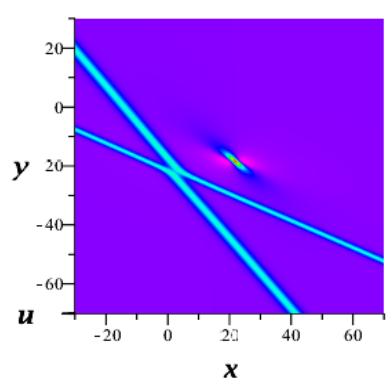

(c) $\mathrm{t}=2$

Figure 3: (Color online) A lump and two line waves never collide with the parameters $\left\{N=4, K_{1}=1+i, K_{2}=1-i, P_{1}=2, P_{2}=2, k_{3}=1, k_{4}=-1, p_{3}=\right.$ $\left.\frac{3}{2}+\frac{3 \sqrt{5}}{10}, p_{4}=-\frac{3}{2}+\frac{3 \sqrt{5}}{10}, \phi_{1}=i \pi, \phi_{2}=i \pi, \phi_{3}=20, \phi_{4}=-20\right\}$. 


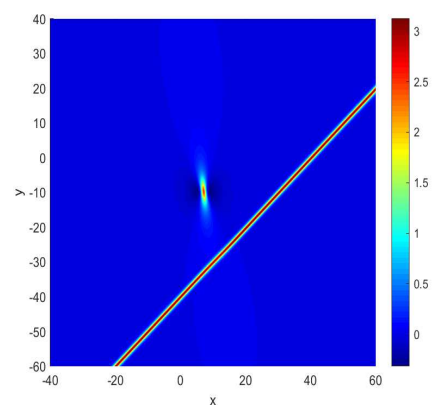

(a) $\mathrm{t}=-10$

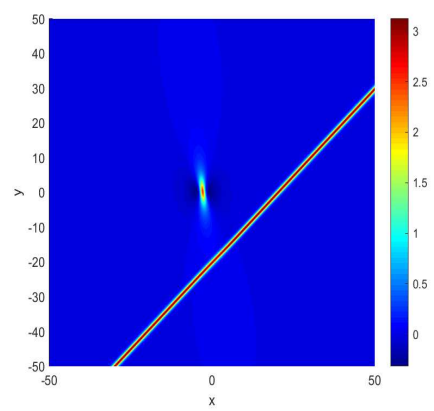

(b) $\mathrm{t}=0$

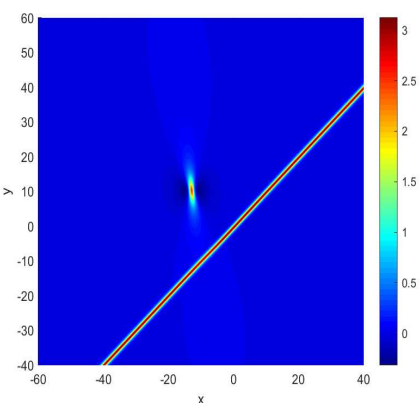

(c) $\mathrm{t}=10$

Figure 4: (Color online) The velocity resonance of a lump wave and a line wave described by Proposition 1 with the parameters $\left\{N=3, K_{1}=\frac{9 \sqrt{19}}{19}+i, K_{2}=\right.$ $\left.\frac{9 \sqrt{19}}{19}-i, k_{3}=-\frac{\sqrt{2 \sqrt{53}+10}}{2}, P_{1}=-\frac{\sqrt{19}}{19}+i, P_{2}=-\frac{\sqrt{19}}{19}-i, p_{3}=\frac{\sqrt{2 \sqrt{53}+10}}{2}, \phi_{1}=i \pi, \phi_{2}=i \pi, \phi_{3}=50\right\}$.

$$
\begin{aligned}
& f_{3}=\theta_{1} \theta_{2}+a_{12}+e^{\left(\lambda_{3} t+\beta_{3}\right)}\left(a_{13} a_{23}+\theta_{1} a_{23}+\theta_{2} a_{13}+\theta_{1} \theta_{2}+a_{12}\right), \\
& \theta_{s}=K_{s} l_{1}+P_{s} l_{2}, \beta_{3}=k_{3} l_{1}+p_{3} l_{2}+\phi_{3}, \quad s=1,2 .
\end{aligned}
$$

Here,

$$
a_{j s}=\left\{\begin{array}{lr}
-\frac{6 K_{j}^{2} k_{s}{ }^{2}\left(K_{j} k_{s}{ }^{3}+K_{j} p_{s}+P_{j} k_{s}\right)}{K_{j}{ }^{2} k_{s}{ }^{6}+2 K_{j}^{2} k_{s}^{3} p_{s}+K_{j} P_{j} k_{s}{ }^{4}+K_{j}^{2} p_{s}{ }^{2}-2 K_{j} P_{j} k_{s} p_{s}+k_{s}{ }^{2} P_{j}^{2}}, & j<s<3 \\
-\frac{6 K_{j}^{2} k_{s}{ }^{2}\left(K_{j} p_{s}+P_{j} k_{s}\right)}{\left(K_{j} p_{s}-P_{j} k_{s}\right)^{2}}, & j=1,2, s \geqslant 3
\end{array}\right.
$$

\section{The new nonlinear superposition of a lump wave and breathers}

It is widely known that soliton molecules and soliton-breather molecules have been extensively studied in many papers, but the velocity resonance of lump wave has rarely been studied. Similarly, by proposing new constraints, novel mixed solutions of lump wave and breathers are obtained. A lump wave can move along the breather wave without collision, or there can be velocity resonance among lump wave and breather waves.

Proposition 2: On the basis of the $N$-soliton solution, if it satisfies the partial long wave limit and module resonance constraints, the new nonlinear superposition of a lump wave and M-breather waves can be derived through the following constraints:

$$
\begin{aligned}
& N=2+2 M, k_{i}=K_{i} \epsilon, p_{i}=P_{i} \epsilon, \phi_{1}=\pi I, K_{1}=K_{2}^{*}, P_{1}=P_{2}^{*}, \epsilon \rightarrow 0, \quad(i=1,2), \\
& k_{1+2 j}=k_{2+2 j}^{*}, p_{1+2 j}=p_{2+2 j}^{*}, \phi_{1+2 j}=\phi_{2+2 j}^{*}, \quad(j=1 . . M) .
\end{aligned}
$$

(i) Lump and breather waves never collide:

$$
\mathfrak{R}\left(\lambda_{s}\right)=\mathfrak{R}\left(-\frac{k_{s}{ }^{6}+5 k_{s}^{3} p_{s}-5 p_{s}^{2}}{k_{s}}+\frac{5 P_{1} P_{2} k_{s}}{K_{1} K_{2}}-\frac{5\left(K_{1} P_{2}+K_{2} P_{1}\right) p_{s}}{K_{1} K_{2}}\right)=0, \quad(s=3 . . N) .
$$

(ii) The velocity resonance of a lump wave and breathers waves:

$$
\left[\frac{5 P_{1} P_{2}}{K_{1} K_{2}}, \frac{-5\left(K_{1} P_{2}+K_{2} P_{1}\right)}{K_{1} K_{2}}\right]^{\mathrm{T}}=\left[-\frac{\mathfrak{R}\left(w_{s}\right) \mathfrak{R}\left(k_{s}\right)}{\left(\mathfrak{R}\left(k_{s}\right)\right)^{2}+\left(\mathfrak{R}\left(p_{s}\right)\right)^{2}},-\frac{\mathfrak{R}\left(w_{s}\right) \mathfrak{R}\left(p_{s}\right)}{\left(\mathfrak{R}\left(k_{s}\right)\right)^{2}+\left(\mathfrak{R}\left(p_{s}\right)\right)^{2}}\right]^{\mathrm{T}}
$$


The novel mixed solutions that we puts forward in Proposition 2 constraint the interaction of lump wave and breather waves. The first is that the lump wave will never collide with the breather wave. Fig.(5) vividly shows the movement of a lump wave over time, and its trajectory is parallel to the breather wave. The second case is the velocity resonance of a lump wave and a breather wave, and they move in the same direction and speed, it is shown in Fig.(6).

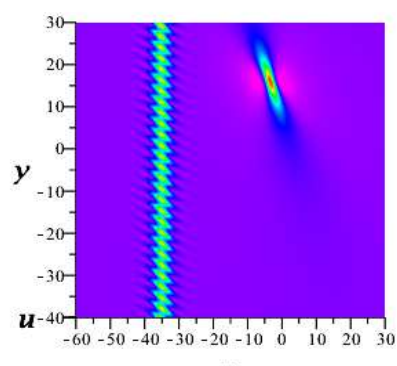

$x$

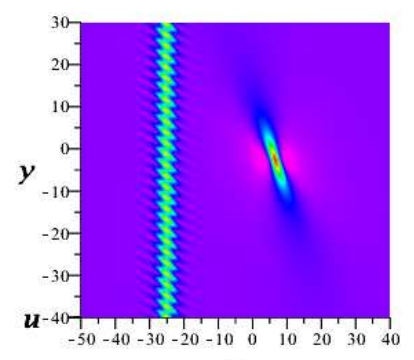

$\boldsymbol{x}$

(b) $\mathrm{t}=0$

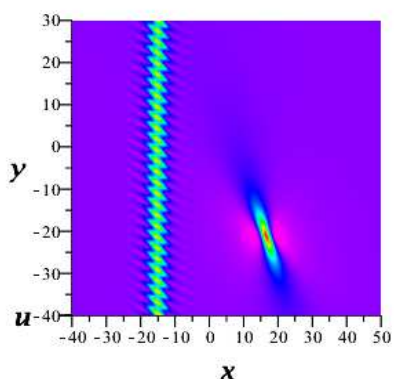

(c) $\mathrm{t}=5$

Figure 5: (Color online) A lump wave and a breather never collide described by Proposition 2 with the parameters $\left\{N=4, K_{1}=\frac{\sqrt{91}}{6}+\frac{i}{2}, K_{2}=\frac{\sqrt{91}}{6}-\frac{i}{2}, k_{3}=\right.$ $\left.\frac{1}{2}+i, k_{4}=\frac{1}{2}-i, P_{1}=\frac{1}{3}+i, P_{2}=\frac{1}{3}-i, p_{3}=i\left(\frac{5}{4}+\frac{\sqrt{91}}{8}\right), p_{4}=-i\left(\frac{5}{4}+\frac{\sqrt{91}}{8}\right), \phi_{1}=i \pi, \phi_{2}=i \pi, \phi_{3}=10, \phi_{4}=10\right\}$.

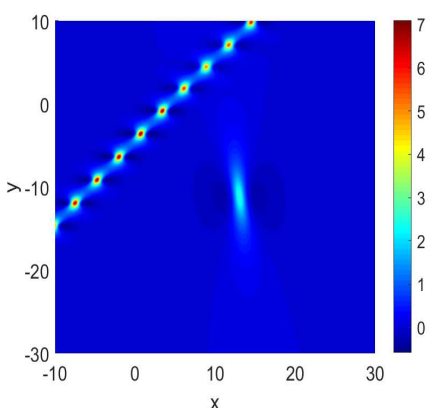

(a) $\mathrm{t}=-10$

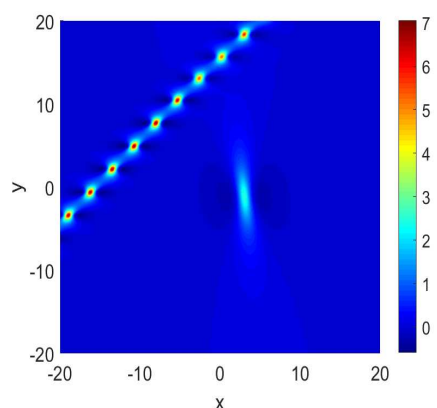

(b) $\mathrm{t}=0$

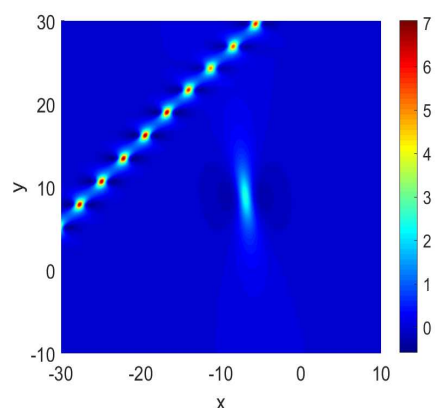

(c) $\mathrm{t}=10$

Figure 6: (Color online) The velocity resonance of a lump wave and a breather wave described by Proposition 2 with the parameters $\left\{N=4, K_{1}=\frac{9 \sqrt{19}}{19}+\right.$ $i, K_{2}=\frac{9 \sqrt{19}}{19}-i, k_{3}=1+\frac{i}{2}, k_{4}=1-\frac{i}{2}, P_{1}=-\frac{\sqrt{19}}{19}+i, P_{2}=-\frac{\sqrt{19}}{19}-i, p_{3}=-1+i\left(\frac{1}{8}+\frac{\sqrt{11}}{2}\right), p_{4}=-1-i\left(\frac{1}{8}+\frac{\sqrt{11}}{2}\right), \phi_{1}=i \pi, \phi_{2}=i \pi, \phi_{3}=$ $\left.15, \phi_{4}=15\right\}$.

Certainly, line waves and breather waves generate breather molecules under the condition of velocity resonance. The breather molecule generated by a line wave and a breather wave of the $(2+1)$-dimensional Sawada-Kotera equation, which is shown in Fig.(7). We also found a very interesting phenomenon, that is, a line wave and a breather wave will generate two breathers in some cases, this phenomenon is vividly shown in Fig.(8), controlling the phase of the line wave, and as a line wave and a breather wave gradually approaching, when $\phi_{3}=3$, two breathers are produced. the reasons for this phenomenon deserve in-depth study.

On the basis of the 3-soliton solutions, restricting parameters to generate breather molecule, the conditions are as follows:

$$
\left[-\frac{w_{3} k_{3}}{k_{3}{ }^{2}+p_{3}{ }^{2}},-\frac{w_{3} p_{3}}{k_{3}{ }^{2}+p_{3}{ }^{2}}\right]^{\mathrm{T}}=\left[-\frac{\mathfrak{R}\left(w_{s}\right) \mathfrak{R}\left(k_{s}\right)}{\left(\mathfrak{R}\left(k_{s}\right)\right)^{2}+\left(\mathfrak{R}\left(p_{s}\right)\right)^{2}},-\frac{\mathfrak{R}\left(w_{s}\right) \mathfrak{R}\left(p_{s}\right)}{\left(\mathfrak{R}\left(k_{s}\right)\right)^{2}+\left(\mathfrak{R}\left(p_{s}\right)\right)^{2}}\right]^{\mathrm{T}},
$$


with

$$
k_{1}=k_{2}^{*}, p_{1}=p_{2}^{*}, \phi_{1}=\phi_{2}^{*} .
$$

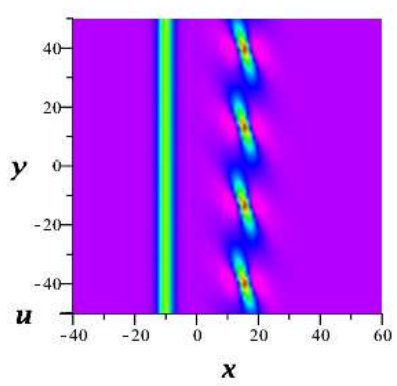

(a) $\mathrm{t}=-10$

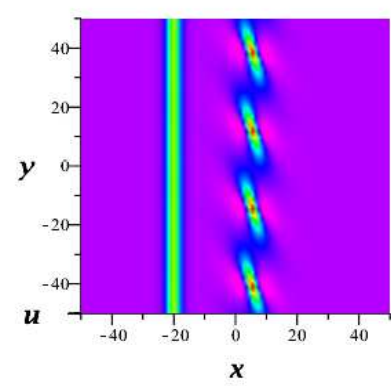

(b) $\mathrm{t}=0$

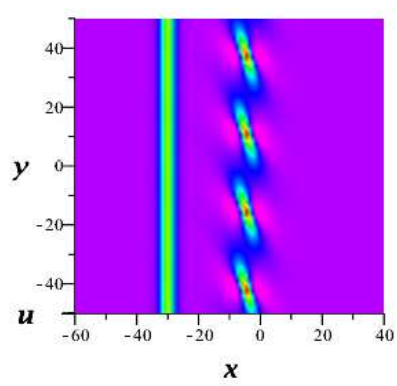

(c) $\mathrm{t}=10$

Figure 7: (Color online) The velocity resonance of a line wave and a breather wave with the parameters $\left\{N=3, k_{1}=\frac{1}{3}+\frac{i}{4}, k_{2}=\frac{1}{3}-\frac{i}{4}, k_{3}=1, p_{1}=\right.$ $\left.i\left(\frac{25}{576}+\frac{5 \sqrt{283}}{432}\right), p_{2}=-i\left(\frac{25}{576}+\frac{5 \sqrt{283}}{432}\right), p_{3}=0, \phi_{1}=0, \phi_{2}=0, \phi_{3}=20\right\}$.

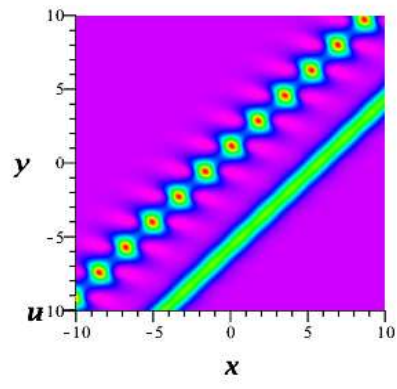

(a) $\phi=-6$

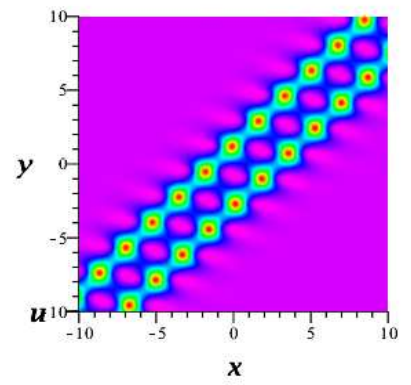

(b) $\phi=2$

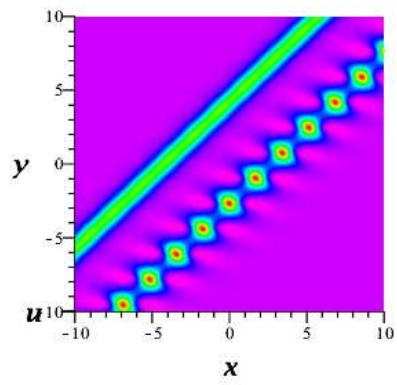

(c) $\phi=10$

Figure 8: (Color online) A line wave gradually approaches the breather to form two breathers with the parameters $\left\{N=3, k_{1}=1+i, k_{2}=1-i, k_{3}=\right.$ $\left.\frac{\sqrt{10+2 \sqrt{37}}}{2}, p_{1}=-1+i\left(1+\frac{\sqrt{70}}{5}\right), p_{2}=-1-i\left(1+\frac{\sqrt{70}}{5}\right), p_{3}=-\frac{\sqrt{10+2 \sqrt{37}}}{2}, \phi_{1}=0, \phi_{2}=0, \phi_{3}=3\right\}$ at $t=0$.

We can also get the velocity resonance of a lump wave, a line wave, and breather waves by comprehensively considering Proposition 1 and Proposition 2, that is, the three keep the same speed and move in the same direction. Based on the $N$-soliton solutions, using partial long-wave limit and module resonance, and satisfying the following conditions Eq.(14), a molecule consisting of a lump, a line wave and a breather waves can be obtained. Once the initial state is determined, the position between them will not be changes over time, it can be seen in Fig.(9), the parameters satisfy the following conditions:

$$
V_{\text {lump }}=V_{\text {line }}=V_{\text {breather }}(s) \text {, }
$$




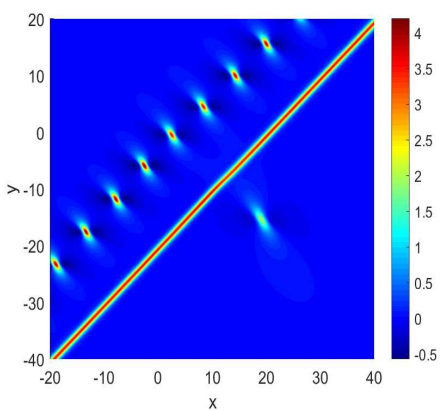

(a) $\mathrm{t}=-2$

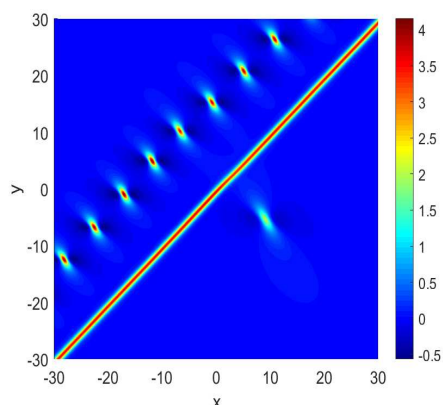

(b) $\mathrm{t}=0$

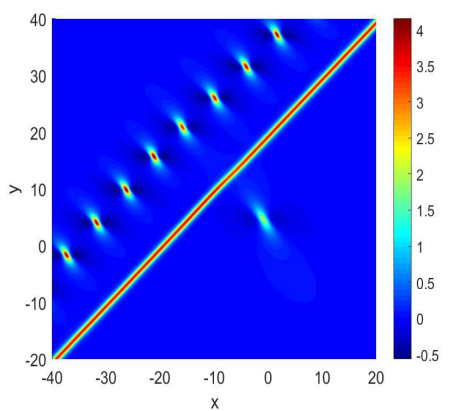

(c) $\mathrm{t}=2$

Figure 9: (Color online) The velocity resonance of a line wave and a breather wave with the parameters $\left\{N=5, K_{1}=3 i, K_{2}=-3 i, k_{3}=\frac{1}{3}+\frac{i}{2}, k_{4}=\right.$ $\frac{1}{3}-\frac{i}{2}, k_{5}=\frac{\sqrt{10+2 \sqrt{85}}}{2}, P_{1}=\frac{3 \sqrt{3}}{2}+\frac{3 i}{2}, P_{2}=\frac{3 \sqrt{3}}{2}-\frac{3 i}{2}, p_{3}=-\frac{1}{3}+i\left(-\frac{23}{72}+\frac{\sqrt{64285}}{270}\right), p_{4}=-\frac{1}{3}-i\left(-\frac{23}{72}+\frac{\sqrt{64285}}{270}\right), p_{5}=-\frac{\sqrt{10+2 \sqrt{85}}}{2}, \phi_{1}=$ $\left.\pi i, \phi_{2}=\pi i, \phi_{3}=5, \phi_{4}=5, \phi_{5}=0\right\}$.

with

$$
\left[\frac{5 P_{1} P_{2}}{K_{1} K_{2}}, \frac{-5\left(K_{1} P_{2}+K_{2} P_{1}\right)}{K_{1} K_{2}}\right]^{\mathrm{T}}=\left[-\frac{w_{3} k_{3}}{k_{3}{ }^{2}+p_{3}{ }^{2}},-\frac{w_{3} p_{3}}{k_{3}^{2}+p_{3}^{2}}\right]^{\mathrm{T}}=\left[-\frac{\mathfrak{R}\left(w_{s}\right) \mathfrak{R}\left(k_{s}\right)}{\left(\mathfrak{R}\left(k_{s}\right)\right)^{2}+\left(\mathfrak{R}\left(p_{s}\right)\right)^{2}},-\frac{\mathfrak{R}\left(w_{s}\right) \mathfrak{R}\left(p_{s}\right)}{\left(\mathfrak{R}\left(k_{s}\right)\right)^{2}+\left(\mathfrak{R}\left(p_{s}\right)\right)^{2}}\right]^{\mathrm{T}}
$$

By extension, the new nonlinear superposition of a lump wave and P-line waves and Q-breather waves can be obtained under the following conditions:

$$
\begin{aligned}
& N=2+P+2 Q, k_{i}=K_{i} \epsilon, p_{i}=P_{i} \epsilon, \phi_{1}=\pi I, K_{1}=K_{2}^{*}, P_{1}=P_{2}^{*}, \epsilon \rightarrow 0, \quad(i=1,2), \\
& k_{2+P+2 j-1}=k_{2+P+2 j}^{*}, p_{2+P+2 j}=p_{2+P+2 j}^{*}, \phi_{2+P+2 j-1}=\phi_{2+P+2 j}^{*}, \quad(j=1 . . Q) . \\
& V_{\text {lump }}=V_{\text {line }}(s)=V_{\text {breather }}(s),
\end{aligned}
$$

There are still many questions about the velocity resonance mechanism of solitons that can be studied, and it is very meaningful in nonlinear dynamical system.

\section{4. conclusion}

According to the velocity formulas of various solitons, this paper studies novel mixed solutions of $(2+1)$-dimensional Sawada-Kotera equation by proposing new constraints. The first is the interaction between a single lump wave and other solitons, on the premise of keeping the motion trajectory of the lump wave parallel to other solitons, whether they can collide forever can be adjusted according to the phase. The second type is the velocity resonance among various solitons, the velocity resonance of line waves and breather waves has been extensively studied by many scholars, but there are few studies on the velocity resonance among lump wave and other waves. This paper enriches this type of new solutions in the field of fluid mechanics. After satisfied with the conditions of velocity resonance, the line wave approaches the breather wave to generate two breather waves, which is also very interesting and the same phenomenon will appear in other integrable systems.

\section{Reference}

[1] Kazimierz Lakomy, Rejish Nath, Luis Santos. Soliton molecules in dipolar Bose-Einstein condensates. Physical Review A, 2012,86013610

[2] Liu Xueming, Yao Xiankun, Cui Yudong. Real-Time Observation of the Buildup of Soliton Molecules. Physical Review Letters, 2018,121 023905

[3] Ma Hongcai, Cheng Qiaoxin, Deng Aiping. Soliton molecules, asymmetric soliton and some novel hybrid solutions for the isospectral BKP equation. Modern Physics Letters B, 2021, 352150174 
[4] Li Yan, Yao Ruoxia, Xia Yarong, Lou Senyue. Plenty of Novel Interaction Structures of Soliton Molecules and Asymmetric Solitons to (2+1)-Dimensional Sawada-Kotera Equation. Communications in Nonlinear Science and Numerical Simulation, 2021, 100105843

[5] Wang Bao, Zhang Zhao, Li Biao. Soliton Molecules and Some Hybrid Solutions for the Nonlinear Schrodinger Equation. Chinese Physics Letters, 2020, 37030501

[6] Zhang Zhao, Yang Shuxin, Li Biao. Soliton Molecules, Asymmetric Solitons and Hybrid Solutions for (2+1)-Dimensional Fifth-Order KdV Equation. Chinese Physics Letters, 2019, 36120501

[7] Lou Senyue. Soliton molecules and asymmetric solitons in three fifth order systems via velocity resonance. Journal of Physics Communications, 2020, 4 041002

[8] Yang Xiangyu, Zhang Zhao, Li Biao. Soliton molecules and dynamics of the smooth positon for the Gerdjikov-Ivanov equation. Chinese Physics B, 2020, 29100501

[9] Yan Zhaowen, Lou Senyue. Special types of solitons and breather molecules for a (2+1)-dimensional fifth-order KdV equation. Communications in Nonlinear Science and Numerical Simulation, 2020,91 105425

[10] Zhang Zhao, Guo Qi, Li Biao, Chen Junchao. A new class of nonlinear superposition between lump waves and other waves for Kadomtsev-Petviashvili I equation. Communications in Nonlinear Science and Numerical Simulation, 2021, 101105866

[11] Tao Yongsheng, He Jingsong. Multisolitons, breathers, and rogue waves for the Hirota equation generated by the Darboux transformation. Physical review. E, 2012, 85026601

[12] Wang Xin, Liu Chong, Wang Lei. Darboux transformation and rogue wave solutions for the variable-coefficients coupled Hirota equations. Journal of Mathematical Analysis and Applications, 2017, 449 1534-1552

[13] Konopelchenko BG, Dubrovsky VG. Some new integrable nonlinear evolution equations in 2+1 dimensions. Physics Letters A, 1984,102 15-17

[14] Chen Qingqing, Qi Zequn, Chen Junchao, Li Biao. Resonant line wave soliton solutions and interaction solutions for (2+1)-dimensional nonlinear wave equation. Results in Physics, 2021, 27104480

[15] Zhang Zhao, Yang Xiangyu, Li Wentao, Li Biao. Trajectory equation of a lump before and after collision with line, lump and breather waves for (2+1)dimensional Kadomtsev-Petviashvili equation. Chinese Physics B, 2019, 28110201

[16] Zhao Zhonglong, He Lingchao. Resonance Y-type soliton and hybrid solutions of a (2+1)-dimensional asymmetrical Nizhnik - Novikov - Veselov equation. Applied Mathematics Letters, 2021, 122107497

[17] Li Jiaheng, Chen Qingqing, Li Biao. Resonance Y-type soliton solutions and some new types of hybrid solutions in the (2+1)-dimensional Sawada-Kotera equation. Communications in Theoretical Physics, 2021, 73045006

[18] Kuo Chunku. Resonant multi-soliton solutions to the (2+1)-dimensional Sawada-Kotera equations via the simplified form of the linear superposition principle. Physica Scripta, 2019, 94085218

[19] An Hongli, Feng Dali, Zhu Haixing. General M-lump, High-order Breather and Localized Interaction Solutions To the (2+1)-dimensional Sawada-kotera Equation. Nonlinear Dynamics, 2019, 98 1275-1286

[20] Li Liuqing, Gao Yitian, Hu Lei, Jia Tingting, Ding Cuicui, Feng Yujie. Bilinear form, soliton, breather, lump and hybrid solutions for a (2+1)-dimensional Sawada - Kotera equation. Nonlinear Dynamics, 2020, 100 2729-2738

[21] Ghanbari Behzad, Kuo Chunku. A variety of solitary wave solutions to the (2+1)-dimensional bidirectional SK and variable-coefficient SK equations. Results in Physics, 2020, 18103266

[22] Wang Wei, Yao Ruoxia, Lou Senyue. Abundant Traveling Wave Structures of (1+1)-Dimensional Sawada-Kotera Equation: Few Cycle Solitons and Soliton Molecules. Chinese Physics Letters, 2020, 37100501

[23] Chen Aihua, Wang Fanfan. Fissionable wave solutions, lump solutions and interactional solutions for the (2+1)-dimensional Sawada-Kotera equation. Physica Scripta, 2019, 94055206

[24] Liu Jianguo. Interaction behaviors for the (2+1)-dimensional Sawada-Kotera equation. Nonlinear Dynamics, 2018, 93 741-747

[25] Huang Lili, Chen Yong. Lump Solutions and Interaction Phenomenon for (2+1)-Dimensional Sawada-Kotera Equation. Communications in Theoretical Physics, 2017, 67 473-478

[26] Zhang Haiqiang, Ma Wenxiu. Lump solutions to the (2+1)-dimensional Sawada-Kotera equation. Nonlinear Dynamics, $2017,872305-2310$

[27] Yao Ruoxia, Li Yan, Lou Senyue. A new set and new relations of multiple soliton solutions of (2+1)-dimensional Sawada-Kotera equation. Communications in Nonlinear Science and Numerical Simulation, 2021, 99105820 


\section{Statements and Declarations}

This work is supported by National Natural Science Foundation of China under Grant Nos. 11775121 and 11435005 , and K.C.Wong Magna Fund in Ningbo University.

The authors have no relevant financial or non-financial interests to disclose.

All data generated or analysed during this study are included in this published article. 